\title{
Gas Evolution in the Planet-Forming Region of Disks
}

\author{
Ilaria Pascucci ${ }^{1,2}$ \\ ${ }^{1}$ Space Telescope Science Institute \\ 3700 San Martin Drive, Baltimore, MD 21218, USA \\ email: pascucci@stsci.edu \\ ${ }^{2}$ Dept. of Physics \& Astronomy, Johns Hopkins University \\ 3400 N. Charles Street, Baltimore, MD 21218, USA \\ email: pascucci@pha.jhu.edu
}

\begin{abstract}
The timescale over which gas-rich disks disperse profoundly affects not only the formation of giant planets but also the habitability of terrestrial planets. In this contributed talk we presented new atomic and molecular diagnostics that can be used to trace the dispersal of gas at disk radii where planets form. We also showed the first observational evidence for photoevaporation driven by the central star and discussed the efficiency of this disk dispersal mechanism.
\end{abstract}

Keywords. accretion disks, line: identification, line: profiles, techniques: spectroscopic, planetary systems: formation, planetary systems: protoplanetary disks

\section{New Gas Line Diagnostics and Disk Photoevaporation}

In spite of its relevance to planet formation, little is known about the gas content of protoplanetary disks at the location where terrestrial and giant planets form. The Spitzer Space Telescope is providing the first view: several atomic and molecular lines, likely probing the planet-forming region, have been detected for the first time toward young sun-like stars. In this contribution, we briefly summarize how newly discovered mid-infrared gas lines can shed light on planet formation and disk dispersal mechanisms:

- $[\mathrm{Ne} \mathrm{II}]$ at $12.81 \mu \mathrm{m}$. This line was first discovered in 2007 in Spitzer spectra of several protoplanetary disks (Pascucci et al. 2007, Lahuis et al. 2007). Models of disks irradiated by stellar X-rays and/or EUV photons (Glassgold et al. 2007, Gorti \& Hollenbach 2009) suggest that $[\mathrm{Ne}$ II] emission arises from the hot $(\sim 5,000 \mathrm{~K})$ disk atmosphere out to about $\sim 15$ AU. Follow-up ground-based high-resolution observations are on the way and confirm the disk origin in sources with no strong jets/outflows (Najita et al. 2009, Pascucci et al. 2009). In a few cases, the line profiles and peak emissions are consistent with disk gas being photoevaporated by the central star (Pascucci \& Sterzik 2009). These results are in agreement with theoretical expectations that EUV-driven photoevaporation occurs at later times in the disk evolution and the mass loss rates are only modest.

- Water and OH transitions. Spitzer spectra of protoplanetary disks around young accreting stars show a forest of water and $\mathrm{OH}$ lines (Carr \& Najita 2008, Salyk et al. 2008). Disks that have already evacuated the inner regions of small dust grains (also called transition disks) lack strong molecular lines in their infrared spectra (Najita et al. in prep.). This could result from low excitation due to low stellar accretion rates or physical depletion of gas in the inner disks, possibly due to an already formed giant planet

- Transitions from organic molecules. Simple organic molecules like $\mathrm{HCN}$ and $\mathrm{C}_{2} \mathrm{H}_{2}$ have been now detected toward many protoplanetary disks (Pascucci et al. 2009). The 


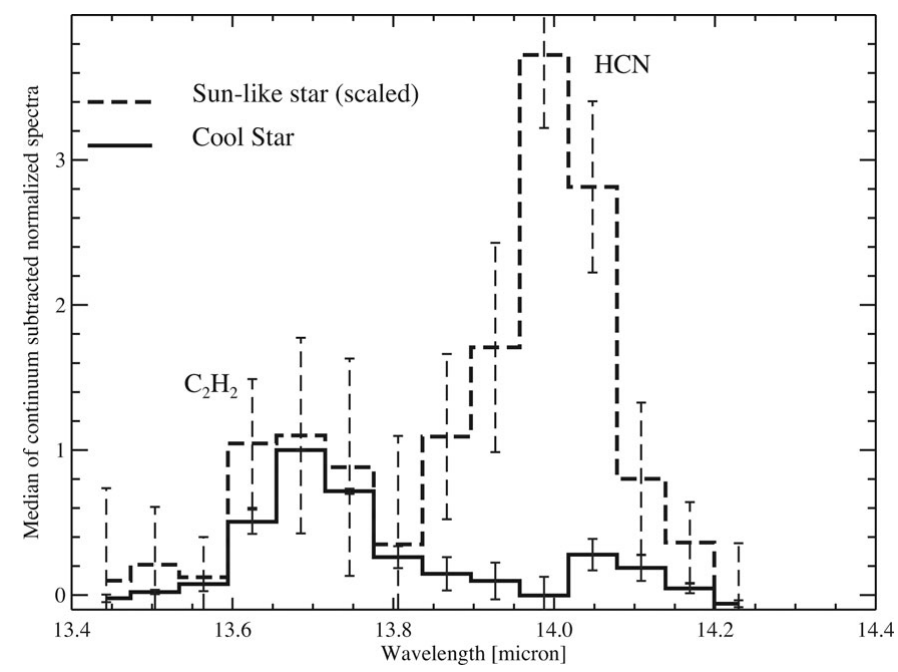

Figure 1. Median of continuum-subtracted and normalized spectra for the T Tauri star (Sun-like star, dashed line) and the very low-mass stars/brown dwarf (Cool star, solid line) samples presenting $\mathrm{C}_{2} \mathrm{H}_{2}$ and/or $\mathrm{HCN}$ emission bands. If brown dwarfs had the same flux ratio of $\mathrm{HCN}$ vs. $\mathrm{C}_{2} \mathrm{H}_{2}$ as the T Tauri stars do, HCN emission would have been easily detected toward them.

detection rate statistics and the line flux ratios of these two molecules show a striking difference between the sample of disks around T Tauri stars and very low-mass stars/brown dwarfs (see Fig. 1), demonstrating a significant underabundance of $\mathrm{HCN}$ relative to $\mathrm{C}_{2} \mathrm{H}_{2}$ in the disk surface of brown dwarfs. These results show how different stellar radiation fields can impact the synthesis of organic molecules and the following chemical disk network.

These recent discoveries with the Spitzer Space Telescope as well as the upcoming Herschel Space Observatory promise a bright future for gas disk studies. Using these different gas line diagnostics it will be possible to provide a definitive statement on the evolution of the gas at disk radii where planet formation takes place, understand whether gas and dust evolve in tandem, and explore whether the early accretion of water on terrestrial planets is a viable scenario.

\section{References}

Carr, J. \& Najita, J. 2008, Science 319, 1504

Glassgold, A., Najita, J., \& Igea, J. 2007, ApJ 656, 515

Gorti, U. \& Hollenbach, D. 2009, ApJ, 690, 1539

Lahuis, F. et al. 2007, ApJ 665, 492

Najita, J. et al. 2009, ApJ 697, 957

Pascucci, I. et al. 2007, ApJ 663,383

Pascucci, I. et al. 2009, ApJ 696, 143

Pascucci, I. \& Sterzik, M. 2009, ApJ 702, 724

Salyk, C. et al. 2008, ApJ (Letters) 676, L49 